\title{
Skeleton Pruning by Contour Partitioning
}

\author{
Xiang Bai ${ }^{1,2}$, Longin Jan Latecki ${ }^{1}$, and Wen-Yu Liu ${ }^{2}$ \\ ${ }^{1}$ CIS Dept., Temple University, Philadelphia, PA 19094, USA \\ \{baixiang, latecki\}@temple.edu \\ ${ }^{2}$ Dept of Electronics \& Information Engineering, Huazhong University of Sci. \& \\ Tech. Wuhan, Hubei. 430074 P.R. China \\ liuwy@hust.edu.cn
}

\begin{abstract}
In this paper, we establish a unique correspondence between skeleton branches and subarcs of object contours. Based on this correspondence, a skeleton is pruned by removing skeleton branches whose generating points are on the same contour subarc. This has an effect of removing redundant skeleton branches and retaining all the necessary visual branches. We show that this approach preserves skeleton topology, does not shift the skeleton, and it does not shrink the remaining branches.
\end{abstract}

Keywords: Skeleton, skeleton pruning, discrete curve evolution.

\section{Introduction}

The skeleton is important for object representation and recognition in different areas, such as image retrieval and computer graphics, character recognition, image processing, and analysis of biomedical images [1]. Skeleton-based representations are the abstraction of objects, which contain both shape features and topological structures of original objects. Due to the importance of the skeleton, many skeletonization algorithms have been developed to represent and measure different shapes. The important factor that constraint the matching of skeletons is the skeleton's sensitivity to object's boundary deformation: little noise or variation of boundary often generates redundant skeleton branches that may disturb the topology of skeleton's graph seriously. For example, the skeleton in Fig. 1(a) has many redundant skeleton branches generated by boundary noise.

The most common approaches to overcome skeleton's instability are based on skeleton pruning, i.e., eliminating redundant skeleton branches. Pruning can be either performed implicitly as a post processing step or implicitly integrated in the skeleton computation. However, none of the existing skeleton pruning methods yields satisfactory results without user interaction. Before we describe the existing skeleton pruning approaches, we characterize desirable properties of skeletons. The skeleton of a single connected shape that is useful for skeleton-based recognition should have the following properties: (1) it should preserve the topological information of the original object; (2) the position of the skeleton should be accurate; (3) it should be stable under small deformations; (4) it should contain the centers of maximal disks, which can be used for reconstruction of original object; (5) it should be invariant under 
Euclidean transformations, such as rotations and translations, and (6) it should represent significant visual parts of objects.

The main goal of this paper is to present a novel skeleton-pruning method that achieves all the above properties. We stress that no existing method can provide a skeleton with all these properties. In addition the proposed method is easy to implement, and can be computed efficiently.

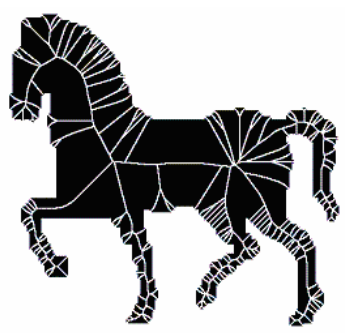

(a)

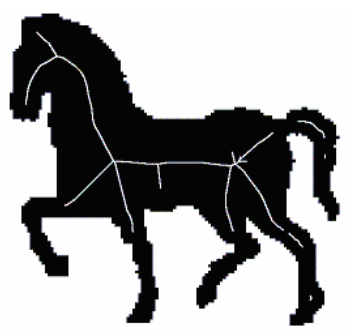

(b)

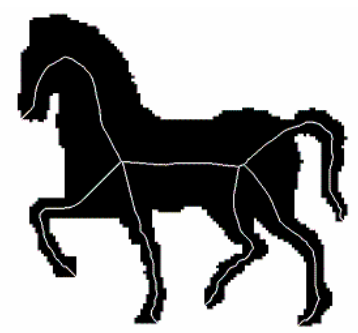

(c)

Fig. 1. The skeleton in (a) has many redundant branches. To remove them, usually skeleton pruning is applied. (b) illustrates the problems of actual pruning approaches (it is generated by a method in [7]). In particular, observe that pruning may change the topology of the original skeleton. (c) illustrates the pruning result of the proposed method that is guaranteed to preserve topology.

Now we present a brief overview of skeletonization and skeleton-pruning approaches. The skeletonization algorithms can broadly be classified into four types: thinning algorithms, e.g., [5, 6]; algorithms based on the Voronoi diagrams, e.g., [2, 8, 22]; algorithms based on distance maps, e.g., [4, 6, 7, 9, 15, 24, 25, 29]; algorithms based on mathematical morphology, e.g., [19-21]. All these algorithms require skeleton pruning, either as a postprocessing step or directly incorporated in the course of computation. There are mainly two ways of pruning methods: (1) based on significance measures assigned to skeleton points [2-4, 16, 22], and (2) based on boundary smoothing before extracting the skeletons $[16,28]$. In particular, curvature flow smoothing still have some significant problems that make the position of skeletons shift and have difficulty in distinguishing noise from low frequency shape information on the boundary [16]. A different kind of smoothing is proposed in [10]. A great progress have been made in the type (1) of pruning approaches that define a significance measure for skeleton points and remove points whose significance is low. Shaked and Bruckstein [16] give a complete analysis and compare such pruning methods. To the common significance measures of skeleton point belong propagation velocity, maximal thickness, radius function, axis arc length, the length of the boundary unfolded. Ogniewicz and Kübler [2] present a few significance measures for pruning hairy Voronoi skeletons without disconnecting the skeletons. Siddiqi et al combine a flux measurement with the thinning process to extract a robust and accurate connected skeleton [20].

All presented methods have several drawbacks. First, many of them are not guaranteed to preserve topology. This is illustrated in Fig. 2, where the skeleton in (d) violates the topology of the input skeleton in (c). This skeleton was obtained by the method in [4]. However, any other method described above would lead to topology 
violation. In particular, all methods presented in [16] (including the method in [2]). Methods described in [16] are guaranteed to preserve topology for simply connected objects (objects with a single contour), but not for objects with more than one contour like the can in Fig. 2. There exist several methods that preserve topology [24, 30] and thinning algorithms (that are usually guaranteed to preserve topology). The topology preserving skeleton obtained by the proposed pruning method is illustrated in Fig. 2(e). Even if the input shape is simply connected many of methods described above are not guaranteed to preserve the original topology (e.g., the skeleton in Fig. 1(b), generated by pruning method in [7]).

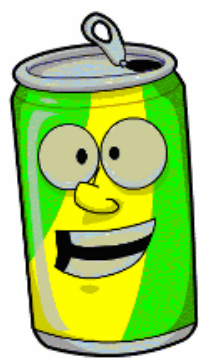

(a)

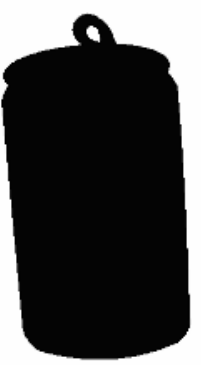

(b)

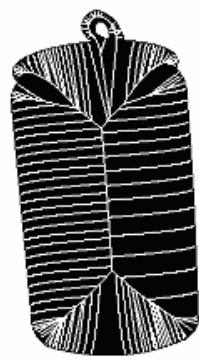

(c)

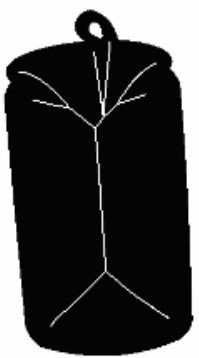

(d)

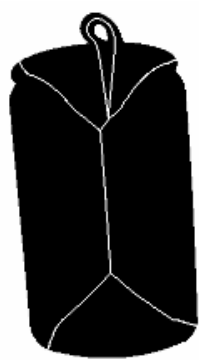

(e)

Fig. 2. (a) The input object. (b) Binary object mask. (c) The initial skeleton. (d) A pruned skeleton obtained by the method in [4]. (e) A pruned skeleton obtained by the proposed method. While skeleton in (d) violates the topology, the proposed method guaranteed to preserve the topology.

The second drawback of all methods described above is that main skeleton branches are shorten to some extent and short skeleton branches are not removed completely, which seriously complicates the structure of the skeletons. These effects are illustrated in Figs. 1(b) and 2(d).

To summarize, although the existing skeleton pruning methods have many drawbacks, they are definitely needed to remove spurious or redundant skeleton branches. The skeleton generating approaches suffer from the fact that a small protrusion on the boundary may result in a large skeleton branch, which is an intrinsic problem of the definition of the skeleton, since the mapping of boundary points to the skeleton points is not continuous. An obvious solution to this problem is to first remove the protrusions on the boundary and then compute the skeleton. As stated above, various smoothing approaches are either applied to the contour or to the distance map before the skeleton is computed. The problem is that isotropic (e.g., Gaussian) as well as anisotropic smoothing only reduces but does not remove the protrusions. A common characteristic of the above approaches is that they displace the boundary points, and consequently displace the location of skeleton points.

\section{Main Ideas of the Proposed Approach}

We propose an approach that completely removes protrusions without displacing the boundary points, and consequently, without displacing the remaining skeleton points. 
Spurious or redundant branches are completely removed while the main branches are not shorten. The main observation of our approach is that it is possible to perform a topology preserving skeleton pruning based on a contour partition into curve segments. Returning to Blum's definition of the skeleton, every skeleton point is linked to boundary points that are tangential to its maximal circle, so called generating points. The main idea is to remove all skeleton points whose generating points all lie on the same contour segment. This works for any contour partition into segments, by some partitions yield better results than other. Fig. 3 illustrated two different pruned skeletons (b) and (c) obtained for the same input skeleton in (a). The pruned skeletons are based on two different partitions into contour segments whose endpoints are marked with dots. For example, removing all skeleton points whose all generating points lie on the contour segment $\mathrm{CD}$ in (b) leads to the removal of the whole lower part of the skeleton. Clearly, the contour partition in (c) leads to a significantly better pruning result than the partition in (b). Thus, in our framework, the question of skeleton pruning is reduced to finding a good partition of the contour into segments. We obtain such partitions with the process of Discrete Curve Evolution (DCE) [11-13].

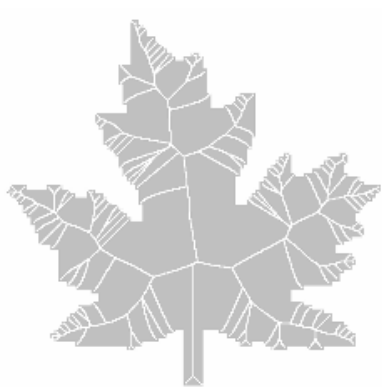

(a)

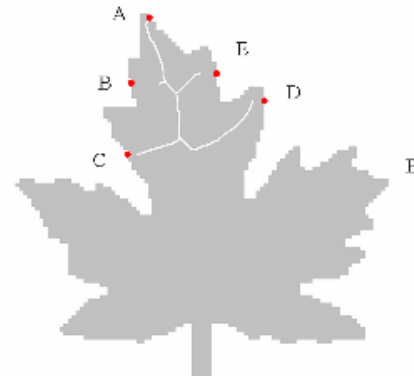

(b)

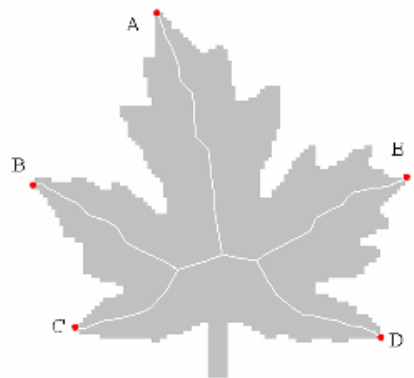

(c)

Fig. 3. Pruning the input skeleton (a) with respect to contour partition induced by five random points on the boundary in (b). The five points in (c) are selected with DCE.

First, observe that every object boundary in a digital image can be represented without loss of information as a finite polygon, due to finite image resolution. The process of DCE is proven experimentally and theoretically to eliminate the noisy points [11-13]. This process eliminates such points by recursively removing polygon vertices with the smallest shape contribution (which are the most likely to result from noise). As the result of DCE, we obtain a subset of vertices that best represent the shape of a given contour. This subset can also be viewed as a partition of the original contour polygon into contour segments defined by consecutive vertices of the simplified polygon. A hierarchical skeleton structure obtained by the proposed approach is illustrated in Fig. 4, where the (red) bounding polygon represents the contour simplified by DCE.

Because DCE can reduce the boundary noise without displacing the remaining boundary points, the accuracy of the skeleton position is guaranteed. The continuity, which implies stability in the presence of noise, of the proposed pruning methods follows from the continuity of the DCE. This means if a given contour and its noisy 
versions are close (measured by Hausdorff distance), the obtained pruned skeletons will be close too. A formal proof of DCE continuity with respect to Hausdorff distance of polygonal curves is given in [18]. Thus, our approach provides a solution to the instability of the classical skeleton pruning algorithms.

The proposed pruning method can be applied to any input skeleton. We only require that each skeleton point is the center of a maximal disk and that the boundary points tangent to the disk (generating points) are given. It is also possible to perform a skeleton growing that includes the proposed pruning method. The main idea is that the pruning is not done in postprocessing (after the skeleton is computed) but is integrated into the skeleton growing process. To implement this idea, we extended the skeleton growing algorithm in [4] based on the Euclidean distance map. First, we select a skeleton seed point as a global maximum of the Euclidean distance map. Then, the remainder of the skeleton points is decided by a growing scheme. In this scheme, the new skeleton points are added using a simple test that examines their eight connected points. During this process, the redundant skeleton branches are eliminated with respect to a given contour partition computed by DCE.
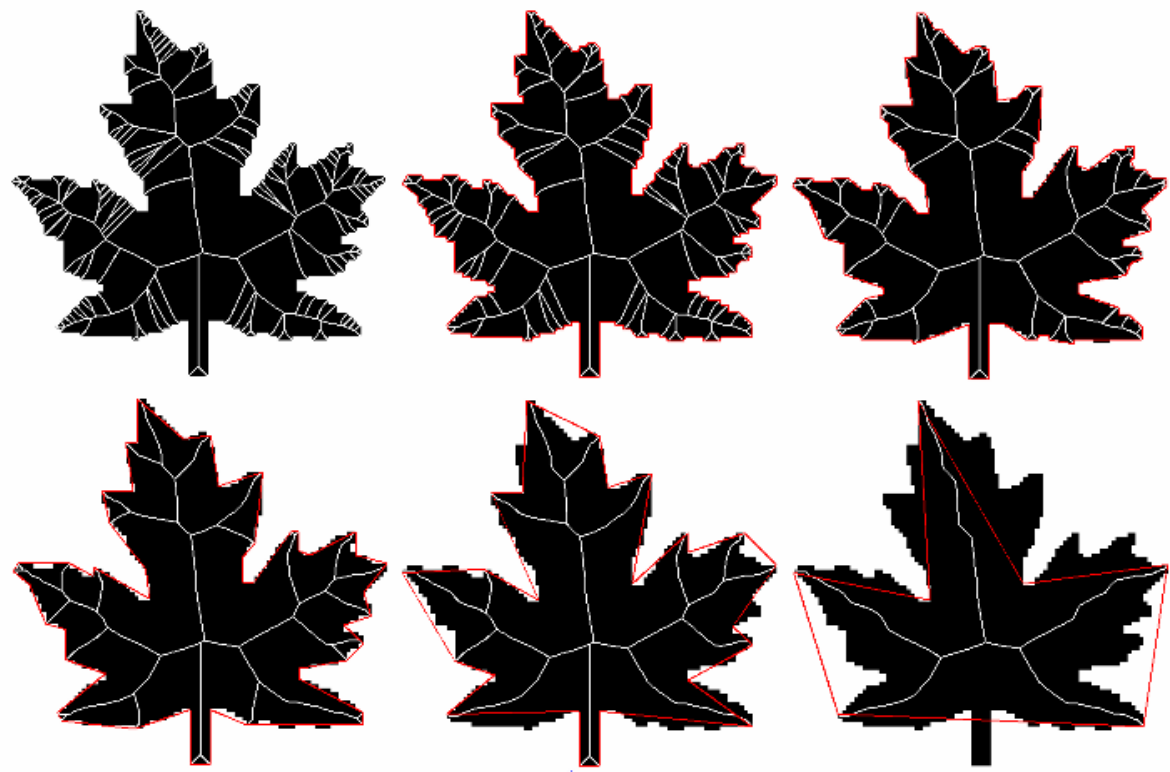

Fig. 4. Hierarchical skeleton of leaf obtained by pruning the input skeleton (top left) with respect to contour segments obtained by the Discrete Curve Evolution (DCE). The outer (red) polylines show the corresponding DCE simplified contours.

Before we formally define the proposed skeleton pruning, we need to characterize planar sets for which we can determine the skeleton. Following [23] we assume that a planer set $D$ is the closure of a connected bounded open subset of $R^{2}$ whose boundary $\partial D$ is composed of a finite number of mutually disjoint simple closed curves. Each simple closed curve in $\partial D$ consists of finite number of pieces of real analytic curves. We further assume in this paper that each simple closed curve is a polygonal curve, 
i.e., the pieces they consist of are line segments. We make this assumption only to simplify some definitions, and we stress that all our results also hold for simple closed curves that consist of finite number of real analytic curves. Observe also that this assumption does not introduce any restriction on object contours in digital images, since each boundary curve in a digital image can be regarded as polygonal curve with vertices being the boundary pixels.

According to Blum's definition of the medial axis [1], the skeleton $S(D)$ of a set $D$ is the locus of the centers of maximal disks. A maximal disk of $D$ is a closed disk contained in $D$ that is interiorly tangent to the boundary $\partial D$ and that is not contained in any other disk in $D$. Observe that each maximal disc must be tangent to the boundary in at least two different points. We denote as Tan(s) the set of the boundary points tangent to the maximal disk $B(s)$ centered at $s \in S(D)$. The points in Tan(s) are called generating points of the skeleton point $s$. Due to our assumption that each boundary curve is a simple closed polygonal curve, Tan(s) is composed of a final number of isolated boundary points, since $\mathrm{B}(\mathrm{s})$ can intersect each boundary line segment in at most one point. (Without this assumption, Tan(s) would be composed of a finite number of isolated contour subarcs.) The degree $\operatorname{deg}(s)$ of $s \in S(D)$ is defined as the cardinality of $\operatorname{Tan}(\mathrm{s})$, i.e., as the number of boundary points tangent to the maximal circle centered at $\mathrm{s}$.

\section{Skeleton Pruning with Contour Partition}

In this section, we formally define the contour partition into contour segments and skeleton pruning based on it. Let the boundary $\partial D$ of a set $D$ be composed of $k$ simple closed curves $C_{1}, \ldots, C_{k}$. Let $x$ and $y$ be two contour points lying on the same simple closed curve $C_{i}$. With $[x, y]$ we denote the shortest closed contour segment (subarc) of $C_{i}$ that connects $x$ and $y$. For simplicity, we assume that $\mathrm{x}$ and $\mathrm{y}$ are positioned on $C_{i}$ so that $[x, y]$ is uniquely determined. With $(x, y)$ we denote the segment $[x, y]$ without the endpoints $\mathrm{x}$ and $\mathrm{y}$ (i.e., the open subarc). (A distinction between open and closed contour segments is unimportant in the digital images, but we need to establish some formal properties in the continuous plane.) A sequence of points $x_{0}, \ldots, x_{n-1}$ on a simple closed curve $C_{i}$ forms a partition of $C_{i}$ if two consecutive segments $\left[\mathrm{x}_{\mathrm{i}}, \mathrm{x}_{\mathrm{i}+1}\right],\left[\mathrm{x}_{\mathrm{i}+1}\right.$, $\left.\mathrm{x}_{\mathrm{i}+2}\right]$ that intersect in $\left\{\mathrm{x}_{\mathrm{i}+1}\right\}$ (the indices are modulo $n$ ), nonconsecutive segments have empty intersection, and $C_{i}$ is the union of these segments. The partition $\Gamma$ of the boundary $\partial D$ is a sequence of sequences that are partitions of the simple closed curves $C_{1}, \ldots, C_{k}$.

Given a partition $\Gamma$ of the boundary $\partial D$ of a simply connected set $D$ (i.e., $\partial \mathrm{D}$ consist of one simple closed curve), the skeleton pruning is defined as removal of all skeleton points $s \in S(D)$ whose all generating points lie in the same open segment $(x, y)$ of partition $\Gamma$. More precisely, the pruned skeleton is composed of all points $s \in S(D)$ such that $\operatorname{Tan}(s)$ is not contained in the same open segment of the partition $\Gamma$. This is a very simple definition of skeleton pruning, and it works with any contour partition. The key issue is to get reasonable partitions. As we will show, DCE provides a very good partition for the pruning. When $D$ is not simply connected (i.e., $\partial \mathrm{D}$ consist of more than one simple closed curve), we additionally need to check whether all skeleton points removed with respect to the same partition segment have their 
generating points on the same simple closed curve $C_{i}$ for some $i=1, \ldots, k$. It can be proven that the topology of pruned skeleton is preserved for pruned skeleton generated by any partition of the contour. The proof is omitted due to the limited space. It will appear in a forthcoming paper. For simplicity of the presentation, we assume in the following definitions that $D$ is simply connected (i.e., $\partial \mathrm{D}$ consist of one simple closed curve).

Now we introduce a contour partition based on Discrete Curve Evolution (DCE). A hierarchical decomposition of the boundary of the set D obtained by DCE is the key component of the proposed skeleton pruning method. Given a skeleton $S(D)$ of a planar shape $D$ and given a DCE simplified contour $D$ given as polygon $P^{k}$ with convex vertices $x_{0}, \ldots, x_{n-1}$, we perform skeleton pruning with respect to the open segments of the partition $\left[x_{i}, x_{i+1}\right]$ (modulo $\mathrm{n}$ ). Thus, we remove all points $\mathrm{s} \in \mathrm{S}(\mathrm{D})$ such that the generating points of $\mathrm{s}, \operatorname{Tan}(s)$, are contained in a single open side of the DCE polygon. Observe that we ignore concave vertices of the DCE simplified polygon.

The simplification of the boundary contour with DCE corresponds to pruning complete branches of the skeleton. In particular, a removal of a single convex vertex $v$ from $P^{n-k}$ to obtain $P^{n-(k+1)}$ by DCE implies a complete removal of the skeleton branch that ends at $v$. We give an example illustrating this fact in Fig. 5. It shows a polygon with seven vertices obtained from the leaf contour by DCE and the skeleton obtained by pruning based on this polygon. Observe that there are only five skeleton branches ending in the five convex vertices of the simplified polygon. The pruned skeleton is computed with respect to the DCE segments $(A, C),(C, D),(D, E),(E, F),(F, A)$. The pruning was applied to the leaf skeleton shown in the first image in Fig. 4. (The skeleton in Fig. 5 is the same as in the last image in Fig. 4.) We can illustrate the main idea of our approach by explaining why the green skeleton branch in Fig. 5 that ends at point $\mathrm{C}$ remained. It remained, since each of its points has maximal disks tangent to points on two different DCE segments, which are contour $\operatorname{arcs}(A, C)$ and $(C, D)$.

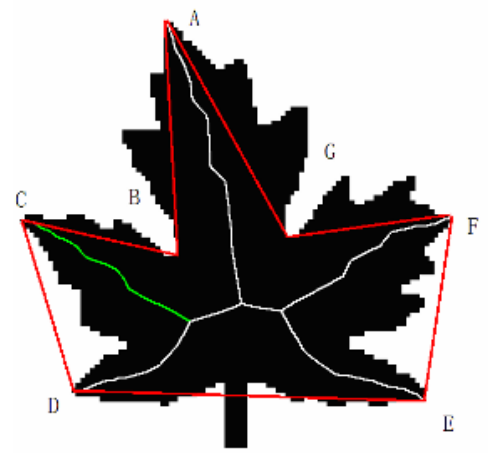

Fig. 5. A simplified polygon with 7 vertexes (in red) and the skeleton obtained based on this polygon. The green skeleton branch remained, since each of its points has generating points on two different arcs $\mathrm{BC}$ and $\mathrm{CD}$ of the original contour.

A very important property of DCE induced contour partition, and every partition that is restricted to vertices of the boundary polygon, is that fact that there is a skeleton branch ending at every partition point. If a partition point that is also a 
polygon vertex $u_{i}$ is deleted in a DCE evolution step, i.e., $u_{i} \in P^{n-k}-P^{n-(k+l)}$, then the arc $\left[u_{i-1}, u_{i+1}\right]$ replaces arcs $\left[u_{i-1}, u_{i}\right],\left[u_{i}, u_{i+1}\right]$ in the contour partition. Consequently, the whole skeleton branch that ends at vertex $u_{i}$ is eliminated by the skeleton pruning.

\section{Experimental Results and Comparison}

\subsection{Stability of Pruning with DCE}

Some results on shapes from MPEG-7 Core Experiment CE-Shape-1 database [27] are showed in Fig. 6. For each shape class, we show pruned skeletons for several objects from the same class. Although the objects differ significantly from each other, the obtained pruned skeletons have the same structures. The final DCE simplified polygons are also showed overlaid on the shapes with red segments. The skeleton pruning is performed with respect to contour partition induced by the vertices of these polygons. In the first row in Fig. 6, the skeletons of the thin and long tails of rats remained complete, which cannot be achieved by other pruning methods, since they may shorten or disconnect the skeleton. Although the camels differ significantly in their shape, all obtained skeletons have similar global structure. The last row of Fig. 6 shows the DCE's stability to the same shapes in different scales.

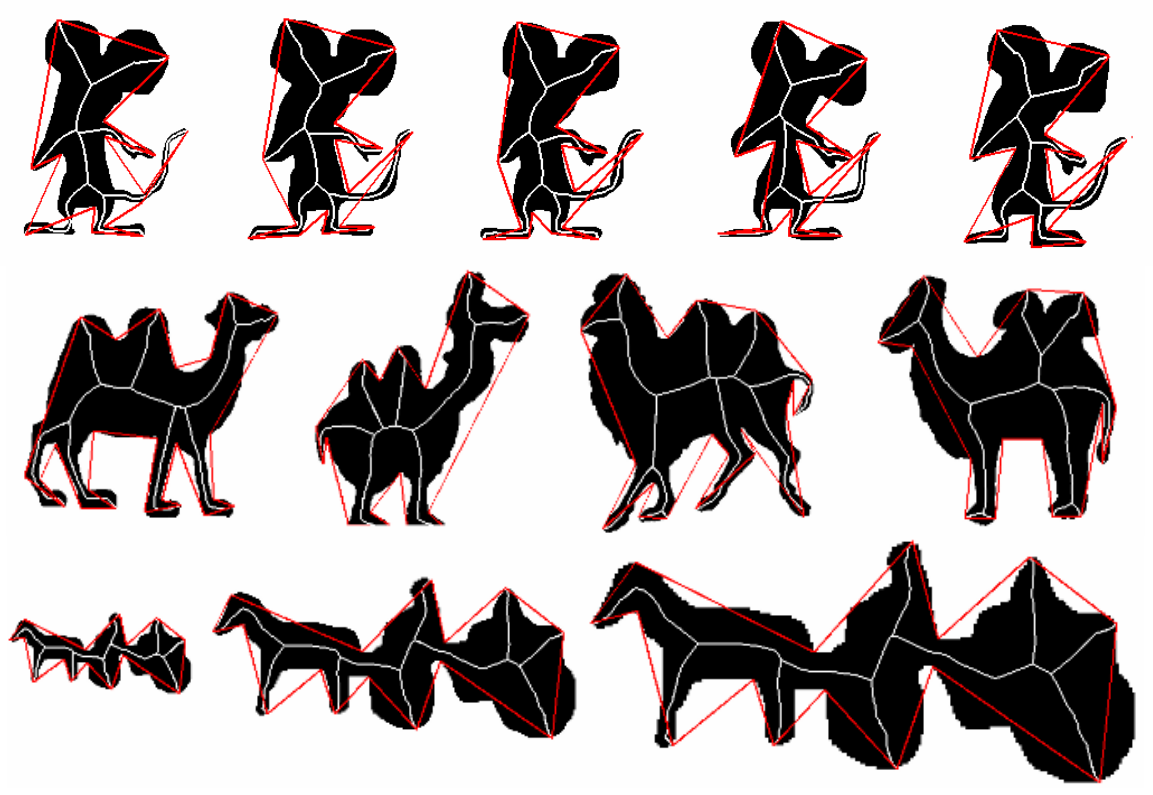

Fig. 6. Our results on Mpeg 7 shape database illustrate extraordinary stability of pruned skeletons in the presence of significant shape variations and deformations 


\subsection{Analysis and Comparison}

In this part, we describe our test results with the proposed approach on several binary shape images with the size $500 \times 500$. All the images tested have significant boundary distortions.

A hierarchy of pruned skeletons is shown for the walking human in Fig. 7. The pruning is preformed with respect to DCE simplified contours with $\mathrm{N}=200,100,50$, 30 , and 12 vertices. We have also shown a hierarchy of pruned skeletons in Fig. 4 above. We can see that the results of our algorithm are in accord with human visual perception. Besides hierarchical and visual property, our skeleton has a unique property: the pruned branches are eliminated completely, i.e., the obtained skeletons are without the presence of remaining half-shortened small, short branches. For example in Fig. 7, each skeleton branch is removed, and no remaining fractions are left.

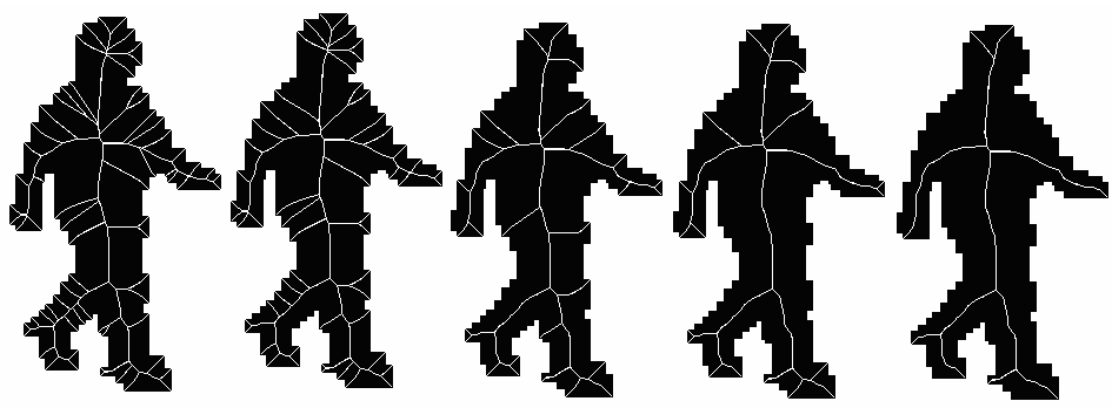

Fig. 7. Hierarchical skeleton of a walking human. The input image is similar to a walking human in [7].

The problem of spurious, half-shortened braches is clearly seen in Fig. 8(a), below, Figs. 1(b) and. 2(d), above, where we see several spurious branches that are not related to any obvious boundary features. Figs. 1 and 2 show a comparison of our method and the method in [4]. As can be clearly seen our method does not suffer from shortening main skeleton branches, and preserves topology of the skeleton. Moreover, the obtained skeletons seem to be in accord with human perception. The result obtained by the method in [4] exhibits clear problems with the skeleton topology and it shortens main skeleton branches in Figs. 1(b) and 2(d).

Our pruning method can also be used in pruning Voronoi skeleton branches. As Voronoi skeleton points are symmetrical to the boundary sample points, the generating boundary points of each skeleton point are known. Fig. 8 shows a comparison of our method to the method by Ogniewicz and Kübler [2]. Fig. 8(b) shows that the proposed approach is able to eliminate all the unimportant branches and still preserve all main structure. Fig. 8(c) shows an application of our method to generate a fixed topology skeleton introduced in Golland and Grimson [7]. It shows that the proposed pruning is not limited to the DCE induced contour partitioning. Once the positions of skeleton's endpoints is estimated along the boundary as in the method in [7], the endpoints induce a partition of the boundary curve, and the fixed 


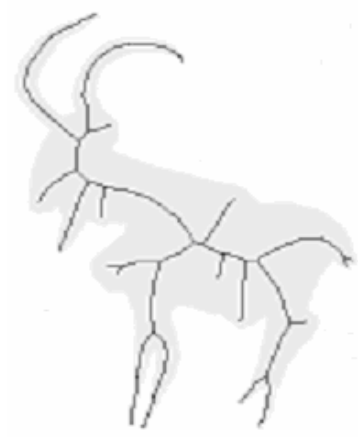

(a)

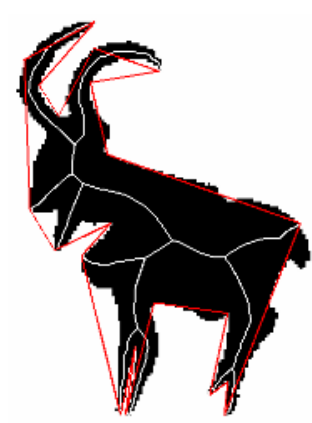

(b)

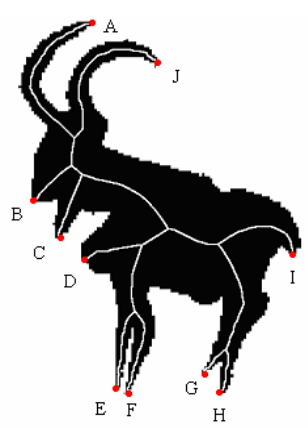

(c)

Fig. 8. Comparison between pruning result in [2] in (a) and our results in (b), and (c) is the result of fixed topology skeleton

topology skeleton can be generated by pruning any skeleton with our method with respect to this partition.

A comparison between a result in [7] and our result is shown in Fig. 9. Fig. 9(a) shows a skeleton obtained by the method in [7], and Fig. 9(b) shows our result induced by the contour partition $(A, B),(B, C),(C, D),(D, E),(E, F)$ marked with the red points, which represent the estimated skeleton endpoints. We can see that the position of our skeleton is more accurate than in (a), since all of our skeleton points are the centers of maximal disks, which are exactly symmetrical to the shape boundary. This is not the case for the fix topology method proposed in [7]. Moreover, compared with [7], only the endpoints need to be estimated; we do not need to estimate the junction points of the skeleton. Another example of a fixed topology skeleton produced with our method is shown in Fig. 8(c).

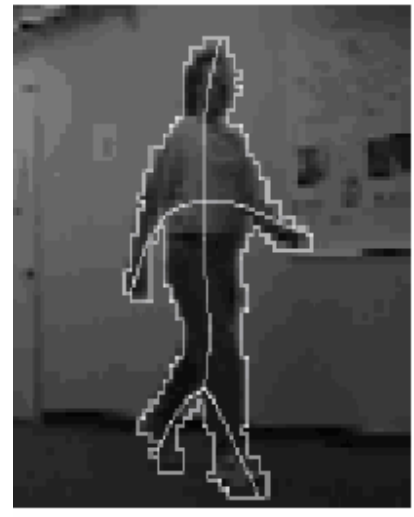

(a)

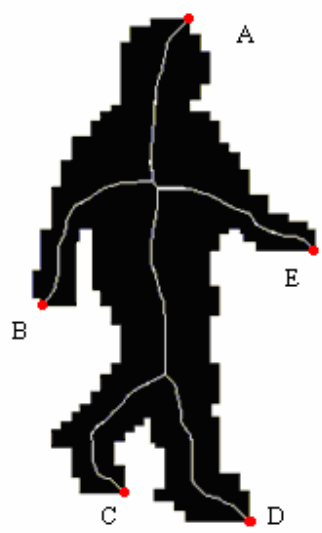

(b)

Fig. 9. Comparison between the fixed topology skeleton in [7] in (a) and our skeleton in (b) 
We omitted here a formal prove that our method is guaranteed to preserve topology. We illustrated this fact in Fig. 2(e) above. Fig. 10 shows another example for a shape with three holes that has total of four contour curves. For comparison, the result of the method in [4] is shown in Fig. 10(b). Fig. 10(c) shows that the proposed approach preserves the original topology. In Fig. 10 (d), the contour partition is only composed of the four boundary curves, i.e., there are no segments on any of the four curves, so that the skeleton points must have their tangent points on the different boundary curves in order to remain.

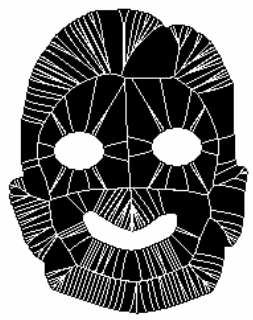

(a)

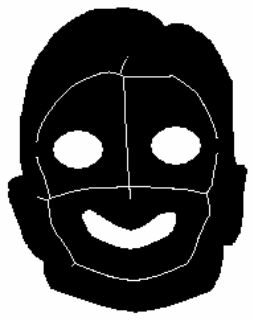

(b)

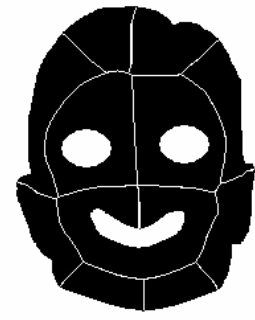

(c)

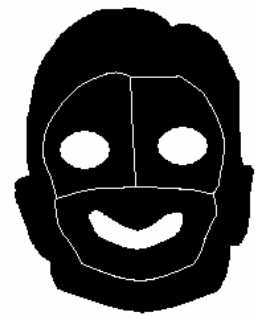

(d)

Fig. 10. (a) The input skeleton. (b) A pruned skeleton obtained by the method in [4] violates the topology. (c,d) Pruned skeletons obtained by the proposed method, which is guaranteed to preserve the topology.

\section{Conclusions and Future Work}

In this paper, we introduce a new skeleton pruning method based on contour partitioning. Any contour partition can be used, but the partitions obtained by Discrete Curve Evolution (DCE) yield very good results. The theoretical properties and the presented experiments demonstrate that the obtained skeletons are in accord with human visual perception and stable, even in the presence of significant noise, and have the same topology as the original skeletons. Thus, we provide a solution to the instability of the classical skeleton algorithms. The stability of skeletons is the key property required to measure shape similarity of objects using their skeletons. Moreover, we have shown that the proposed approach never produces spurious branches, so common to the known skeleton pruning methods, and that the proposed pruning method does not displace the skeleton points. Consequently, all skeleton points are centers of maximal disks. We have proven that our pruning method is guaranteed to preserve topology. The proof will appear in a forthcoming paper.

\section{References}

1. Blum, H.: Biological Shape and Visual Science (Part I). J. Theoretical Biology 38 (1973) 205-287

2. Ogniewicz, R.L., Kübler, O.: Hierarchic Voronoi skeletons, Pattern Recognition 28(3) (1995) 343-359 
3. Malandain, G., Fernandez-Vidal, S.: Euclidean skeletons. Image and Vision Computing 16 (1998) 317-327

4. Choi, W.-P., Lam, K.-M., Siu, W.-C.: Extraction of the Euclidean skeleton based on a connectivity criterion. Pattern Recognition 36 (2003) 721-729

5. Pudney, C.: Distance-Ordered Homotopic Thinning: A Skeletonization Algorithm for 3D Digital Images. Computer Vision and Image Understanding 72(3) (1998) 404-413

6. Leymarie, F., Levine, M.: Simulating the grassfire transaction form using an active Contour model. IEEE Trans. PAMI 14(1) (1992) 56-75

7. Golland, P., Grimson, E.: Fixed topology skeletons. In CVPR, Vol. 1 (2000) 10-17

8. Mayya, N., Rajan, V.T.: Voronoi Diagrams of polygons: A framework for Shape Representation. Proc. of the IEEE CVPR (1994) 638-643

9. Ge, Y., Fitzpatrick, J.M.: On the Generation of Skeletons from Discrete Euclidean Distance Maps. IEEE Trans. PAMI 18(11) (1996) 1055-1066

10. Gold, C.M., Thibault, D., Liu, Z.: Map Generalization by Skeleton Retraction. ICA Workshop on Map Generalization, Ottawa, August 1999

11. Latecki, L.J., Lakämper, R.: Convexity Rule for Shape Decomposition Based on Discrete Contour Evolution. Computer Vision and Image Understanding (CVIU) 73 (1999) 441454

12. Latecki, L.J., Lakamper, R.: Polygon evolution by vertex deletion. Proc. of Int. Conf. on Scale-Space, volume LNCS 1682 (1999)

13. Latecki, L.J., Lakamper, R.: Shape similarity measure based on correspondence of visual parts, IEEE Trans. Pattern Analysis and Machine Intelligence (PAMI) 22(10) (2000) $1185-1190$

14. Latecki, L.J., Lakamper, R.: Application of planar shape comparison to object retrieval in image databases. Pattern Recognition 35(1) (2002) 15-29

15. Borgefors, G.: Distance transformations in digital images. Computer Vision, Graphics and Image Processing 34(3) (1986) 344-371

16. Shaken, D., Bruckstein, A.M.: Pruning Medial Axes. Computer Vision and Image Understanding 69(2) (1998) 156-169

17. Dimitrov, P., Damon, J.N., Siddiqi, K.: Flux Invariants for Shape. CVPR (2003)

18. Latecki, L.J., Ghadially, R.-R., Lakämper, R., Eckhardt, U.: Continuity of the discrete curve evolution. Journal of Electronic Imaging 9(3) (2000) 317-326

19. Dimitrov, P., Phillips, C., Siddiqi, K.: Robust and Efficient Skeletal Graphs. In CVPR (2000) 1417-1423

20. Siddiqi, K., Bouix, S., Tannenbaum, A.R., Zucker, S.W.: Hamilton-Jacobi Skeletons. International Journal of Computer Vision 48(3) (2002) 215-231

21. Vasilevskiy, A., Siddiqi, K.: Flux Maximizing Geometric Flows. IEEE Trans. PAMI 24(12) (2002) 1565-1578

22. Brandt, J.W. Algazi, V.R.: Continuous skeleton computation by Voronoi diagram. Comput. Vision, Graphics, Image Processing 55 (1992) 329-338

23. Choi, H.I., Choi, S.W., Moon, H.P.: Mathematical Theory of Medial Axis Transform. Pacific Journal of Mathematics 181(1) (1997) 57-88

24. Arcelli, C. Sanniti di Baja, G.: Euclidean skeleton via center of maximal disk extraction. Image and Vision Computing 11 (1993) 163-173

25. Kimmel, R., et al.: Skeletonization via Distance Maps and Level Sets. CVIU: Comp. Vision and Image Understanding 62(3) (1995) 382-391

26. Sebastian, T.B., Klein, P.N., Kimia, B.B.: Recognition of shapes by editing their shock graphs. IEEE Trans. PAMI 26(5) (2004) 550-571 
27. Latecki, L.J., Lakamper, R., Eckhardt, U.: Shape Descriptors for Non-rigid Shapes with a Single Closed Contour. Proc. CVPR (2000)

28. Pizer, S.M., Oliver, W.R., Bloomberg, S.H.: Hierarchial shape description via the multiresolution symmetric axis transform. IEEE Trans. PAMI 9 (1987) 505-511

29. Borgefors, G., Ramella, G., Sanniti di Baja, G.: Hierarchical decomposition of multiscale skeletons. IEEE Trans. PAMI 13(11) (2001) 1296-1312

30. Sanniti di Baja, G.: Well-shaped, stable and reversible skeletons from the $(3,4)$-distance transform. Visual Communication and Image Representation 5 (1994) 107-115 